COMUNICACIÓN CIENTÍfICA IX CONGRESO NACIONAL DE LA SOCIEDAD CIENTÍFICA ESPAÑOLA DE ENFERMERÍA - SCELE. Mayo de 2018. UNIVERSIDAD DE ALICANTE.

\title{
DESCONTAMINACIÓN DIGESTIVA: IMPLEMENTANDO LA SEGURIDAD DEL PACIENTE CRÍtICO CON VENTILACIÓN MECÁNICA (VM)
}

MA Antón-Latour

Enfermera de cuidados críticos en UCI Quirúrgica del Hospital General Universitario de Elche; Coordinadora de Calidad de la UCI Quirúrgica del Hospital General Universitario de Elche

E-mail: masunanton@gmail.com 


\section{PALABRAS CLAVE:}

Seguridad del paciente, Neumonía, Ventilación mecánica, Desconta-minación selectiva del tubo digestivo (DDS).

\section{Objetivo general:}

- Promover y mejorar la cultura de seguridad del paciente en la UCI Quirúrgica(UCIQ). Objetivo específico:

- Reducir la tasa de densidad de incidencia (DI) de la neumonía asociada a ventilación mecánica (NAVM) en nuestra unidad a menos de 5,77 episodios de NAVM por 1.000 días de VM, que representa una reducción del 66\% respecto a la tasa media estatal de los años 2000-2008 en las Unidades de Críticos españolas y una reducción del 39,5\% con respecto a la de los años 2009-2013.

2. Métodos: Estudio longitudinal de tipo prospectivo, estructurado en:

1) Implantación en la UCIQ del Hospital General Universitario de Elche del protocolo de cuidados para prevenir la NAVM (enero 2015), mediante la aplicación de un paquete de medidas básicas (de obligado cumplimiento) y otro de medidas específicas (altamente recomendables).

2)Se aplica a todos los pacientes intubados o traqueostomizados, ingresados en la UCIQ y sometidos a VM.

3)Incorporación de la medida específica altamente recomendable (IA)

"Descontaminación selectiva del tubo digestivo (completa u orofaríngea)" (enero 2017)

Eliminación de los microorganismos potencialmente patógenos, mediante la administración de una pasta oral / faríngea y una suspensión digestiva durante todos los días de VM, y antibióticos sistémicos durante los dos primeros días.

4)La intervención para la prevención de NAVM, como vehículo para alcanzar la Neumonía Zero, es una de las dos actividades complementarias e igualmente importantes, según el Plan de Seguridad Integral (PSI), el cual persigue promover y fortalecer la cultura de seguridad en el trabajo diario en las UCIs, y que ha demostrado ser esencial para la consecución de los objetivos del proyecto Bacteriemia Zero (BZ), de manera que no se puede contemplar aplicar el paquete de medidas sin aplicar el PSI. Los equipos que participan en el proyecto de reducción de NAVM se comprometen al cumplimiento de los dos brazos de la intervención.

5)Para el análisis de resultados, se incluyen en el estudio todos los pacientes a partir de las 48 horas con VM en UCIQ, y todos los casos de NAVM identificados en UCIQ y diagnosticados hasta las siguientes 48 horas después de su traslado a otra unidad. Dicho análisis se realiza anualmente por el servicio de Medicina Preventiva del HGU de Elche.

3. Implicaciones para la práctica clínica: La reducción de la tasa DI de NAVM supondrá una disminución de la estancia en UCIQ, de consumo de antibióticos, del coste hospitalario, y de la mortalidad que lleva asociada esta complicación.

Se amplia la cultura de seguridad del paciente, con una medida de bajo coste: DDS

\section{Limitaciones}

La recogida de datos por parte de medicina preventiva se interrumpe durante el periodo vacacional. 